\title{
Cosmic ray intensity variations in the 24th solar cycle observed by LAAS experiments
}

\author{
Naoki Wada ${ }^{a *}$, Atsushi lyono $^{\dagger b}$, Hiroki Matsumoto $^{c}$, Kazuhide Okei $^{c}$, Shuhei Tsuji ${ }^{c}$ \\ Soji Ohara ${ }^{d}{ }$ Nobuaki Ochi $^{e}$, Nobusuke Takahashi $^{f}{ }$, Takao Nakatsuka ${ }^{g}$, Isao \\ Yamamoto $^{h}$ and Saya Yamamoto ${ }^{a}$ \\ ${ }^{a}$ Graduate School of Science, Okayama University of Science, \\ 1-1 Ridai-cho, Kita-ku, Okayama-city, Okayama 700-0005, Japan \\ ${ }^{b}$ Dept. of Fundamental Science, Faculty of Science, Okayama University of Science, \\ 1-1 Ridai-cho, Kita-ku, Okayama-city, Okayama 700-0005, Japan \\ ${ }^{c}$ Kawasaki Medical School, \\ 577 Matsushima, Kurashiki-city, Okayama 701-0192, Japan \\ ${ }^{d}$ Department of Informatics, Naragakuen University, \\ Ikomagun-Nara, 6368503, Japan \\ ${ }^{e}$ Faculty of Business Administration, Toyo University, \\ 5-28-20 Hakusan, Bunkyo-ku, Tokyo 112-8606, Japan \\ ${ }^{f}$ Department of Advanced Physics, Faculty of Science and Technology, Hirosaki University, \\ 1 Bunkyo-cho, Hirosaki-shi, Aomori-ken,036-8560, Japan \\ g Okayama Shoka University, \\ 2-10-1Tsushima Kyomachi, Kita-ku, Okayama-city,Okayama 700-8601, Japan \\ ${ }^{h}$ Department of Information and Computer Engineering, Faculty of Engineering, Okayama \\ University of Science, \\ 1-1 Ridai-cho, Kita-ku, Okayama-city, Okayama 700-0005, Japan \\ E-mail: iyonoddas.ous.ac.jp, h.matsumoto@med.kawasaki-m.ac.jp \\ okei@med.kawasaki-m.ac.jp tsuji@med.kawasaki-m.ac.jp \\ ohara@nara-su.ac.jp ochi@hikarigai.net taka@cc.hirosaki-u.ac.jp \\ nakatuka@olive.plala.or.jp i-yama@zpost.plala.or.jp \\ s15sm08dous.jp
}

In Large Area Air Shower experiments, one of EAS arrays is deployed on the second floor of four stories building in the campus of Okayama University of Science to limit the zenith angle acceptance of muon detection. This system provide EAS muon intensities from zenith solid angles. To compare these intensities with solar activities such as flares, CME and magneticstorms, LAAS data have been analyzed. Faster data acquisition with $\mathrm{Ha} / \mathrm{CaK}$ solar telescope have also been carried out. In this presentation, cosmic ray variations for four years since 2011 are going to be reported as well as fast image data.

The 34th International Cosmic Ray Conference,

30 July- 6 August, 2015

The Hague, The Netherlands

* current affiliation: TOSHIBA MEMORY SYSTEMS Co.,Ltd.

${ }^{\dagger}$ Speaker. 


\section{Introduction}

Below $\mathrm{TeV}$ energies of cosmic rays, the variation in solar activity yields several physical impacts to the heliosphere, and consequently the modulations of galactic cosmic ray flux observed at the earth orbit arose from the strong turbulence of the interplanetary magnetic field due to solar incidents such as solar flares,filament eruptions and coronal mass ejections. The Forbush decreases [2] are the most significant suppressions. On the other hand, the neutron monitor experiments [7, 6] systematically have registered the sporadic and long term variabilities of the cosmic ray flux since 1950s. The sudden increases of neutron multiplicity in several milliseconds [3, 6] have also observed in their network observations, called as solar neutron events. In this case, solar neutrons were produced when the high energy solar particles accelerated in the solar flares, strike the solar photosphere below its transition layers. Because the target particle density decrease by about three order at this transition layer $\left(5 \times 10^{-16}\right.$ to $\left.2 \times 10^{-13} \mathrm{~g} / \mathrm{cm}^{3}\right)$, hadronic interactions of high energy ions in solar flare with the particles in the photosphere might occur simultaneously.

In the paper, we report the results of the galactic cosmic ray intensity variations after some solar events such as solar flares and CMEs observed by LAAS EAS arrays [4 5]. And we carried out $\mathrm{CaK}$ telescope observations with the high exposure rates to search the correlations of the cosmic ray flux and the neutron monitor networks.

\section{Apparatus}

\subsection{LAAS EAS arrays}

The LAAS experiments [4] have maintained eight EAS arrays scattered over in Japan since 1996, with GPS-synchronized time stamp system of which accuracy is 1 microsecond, to investigate the cause of simultaneous EAS incidences detected more than $100 \mathrm{~km}$ baseline of EAS arrays. One of physics goals is to study the photo-disintegration effects of very high energy cosmic ray nuclei above $10^{17} \mathrm{eV}$ with solar photons in the interplanetary space before cosmic ray nuclei arriving at the earth' atmosphere, which was proposed as the cue of cosmic ray composition studies above knee energies in 1950s by Gerasimova and Zatsepin. As part of LAAS arrays, the four sets of compact EAS arrays are located at Okayama University of Science (OUS), Okayama city, Japan, under a mean atmospheric overburden of $1036 \mathrm{~g} / \mathrm{cm} 2$. The EAS array OUS4 [5] is installed in order to restrict the observation of EAS zenith angles.

The EAS arrays typically consists of eight plastic scintillation counters in as shown in Figure 11 and each counter is equipped with a scintillator of which size and thickness are $50 \mathrm{~cm} \times 50 \mathrm{~cm}$ and $5 \mathrm{~cm}$ respectively, and a fast photomultiplier. The detectors are deployed on the rooftop of the building in the university campus and located over an area of about $200 \mathrm{~m}^{2}$. The experiment data are acquired by a TDC, a ADC, and a shift register when receiving the trigger request signal from the coincidence module. The trigger request signal is generated when more than 3 detectors are hit within 2.5 microsecond time window and sets the trigger time to zero second. EAS particle signals within \pm 2.5 microsecond from the trigger time of zero second were recorded by the shift register system. The time stamp of triggered events is also obtained by a GPS timing module. This module maintains $10 \mathrm{MHz}$ oscillator synchronized with GPS 1 pps signal within 1microsecond accuracy and provides the EAS arrival timing in Universal Time with an accuracy of 1 microsecond. Because 
correlations between arrival times and directions of EAS over the distance of more than 100km are searched in LAAS experiments, the accuracy is enough to separate the EAS events.

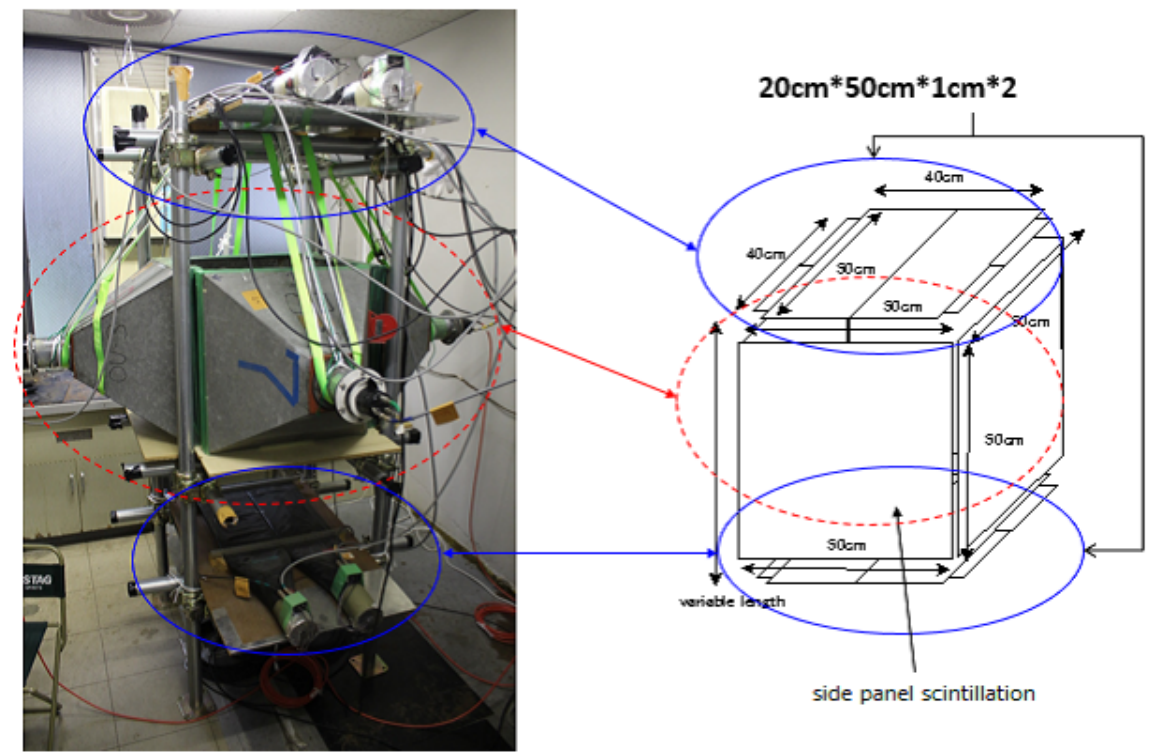

Figure 1: The photograph and schematic view of the OUS4 array.

The OUS4 [5] has been constructed in the second floor of the four stories building, shown in Figure 1 At the top and the bottom layer of the OUS4, four scintillation counters, of which size and thickness are $20 \mathrm{~cm} \times 50 \mathrm{~cm}$ and $1 \mathrm{~cm}$, respectively, are used in order to generate a trigger signal. And at the side panels $50 \mathrm{~cm} \times 50 \mathrm{~cm} \times 5 \mathrm{~cm}$ thickness scintillation counters are also installed, as shown in Figure 1 Those are needed to eliminate the EAS events of which zenith angles are more than 25.6 degree. We designed the experimental set up of detectors at the OUS4 in order to select the EAS events whose the zenith angle should be less than 25.6 degree, among uniformly incident EAS events. The instrument of the OUS4 consists of top and bottom scintillation counters in coincidence, separated by $1350 \mathrm{~mm}$. The maximum zenith angle that will trigger the OUS4 depends on the diagonal length of the set of scintillation counters $640 \mathrm{~mm}$ and separated length of $1350 \mathrm{~mm}[5]$.

\section{2 $\mathrm{H} \alpha$ and CaK telescope imaging}

To observe the photo-spheric granulation and sunspot in the solar photosphere, CaK line filtered imaging telescope shown in Figure 2(Lunt CaK blocking filter, $393.4 \mathrm{~nm}$ with the FWHM of 2.4 angstrom) and CCD camera (Point Gray, Blackfly PGE05S2M, SONY ICX693 CCD sensor ) have been used. Its focal length and F-value are $550 \mathrm{~mm}$ and F/6.6 respectively. To detect the intensity variation just after solar flare events in the photosphere, image data of which pixel size is $800 \times 600$ pixels, are acquired in every two seconds. Although the focal length and aperture size of our telescope do not provide high resolution imaging, second order time-resolved imaging could enable to explore the photosphere events accompanied with solar flare events since neutron bursts 


\begin{tabular}{|l||c|}
\hline & Period \\
\hline CaK/H $\alpha$ imaging & $2013 / 09 / 11-11 / 26,2014 / 09 / 16-2015 / 01 / 09$ \\
\hline OUS4 & $2008 / 05 / 01-2012 / 12 / 31$ \\
\hline OUS4 & $2014 / 08 / 26-2015 / 01 / 04$ \\
\hline
\end{tabular}

Table 1: Data period of observations with $\mathrm{h} \alpha$, CaK and EAS systems.

had been observed in the several millisecond time intervals. The monochromatic imaging device have recorded the $\mathrm{CaK}$ line filtered photosphere if the weather permits.

To observe the solar chromosphere brightening associated with the solar flares, we have used $\mathrm{H} \alpha$ telescope (Coronado Solar Max 60 with double stacked Etalon filters) and CMOS sensors for imaging devices(Canon 60D without $\mathrm{H} \alpha$ filter) as shown in Figure 2 Double stacked Etalon filter provides the FWHM of about 0.4 angstrom at $656 \mathrm{~nm}$ wavelength. Its focal length and F-value are 400mm and F6/6.6 respectively. Data acquisition have been carried out on every five seconds with about 1/200 second exposure and ISO100 equivalent gain in the raw image format.

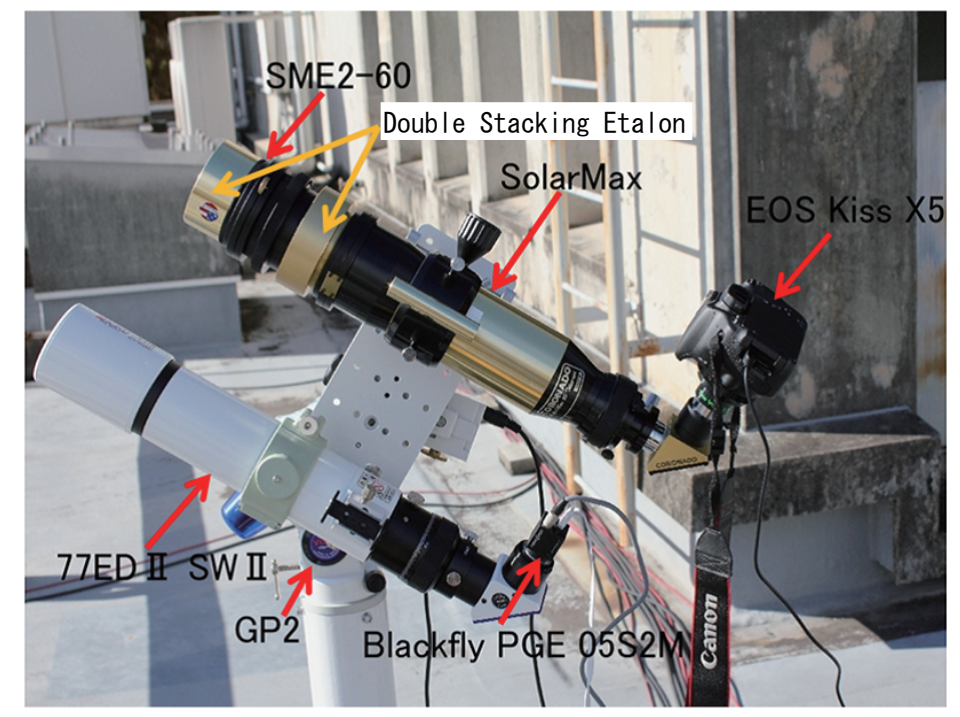

Figure 2: $\mathrm{H} \alpha$ and $\mathrm{CaK}$ filtered imaging telescopes with $\mathrm{CCD}$ and CMOS sensors

\section{Data Analysis}

The data periods of CaK imaging observations and EAS observations are summarized in Table 11 The solar flare activities occurred int these observations are also listed in Table 2 The OUS4 array have moved to the other laboratory after January 2013 and we have restarted it in the middle of 2014 at the second floor of 5 story building.

\section{Results}

One of our purpose is to search the light curve variation of the solar photosphere during the so- 
Table 2: Flare list in the period of this analysis observed by GOES satellite

\begin{tabular}{|c|c|c|c|}
\hline \multirow{2}{*}{ period } & \multicolumn{3}{|c|}{ Flare magnitude } \\
\cline { 2 - 4 } & $\mathrm{M}$ & $\mathrm{X}$ & $\mathrm{A}-\mathrm{X}$ \\
\hline $2011 / 02 / 08 \sim 02 / 22$ & 11 & 1 & 141 \\
\hline $2011 / 03 / 02 \sim 03 / 20$ & 15 & 1 & 159 \\
\hline $2011 / 11 / 01 \sim 11 / 14$ & 10 & 1 & 100 \\
\hline $2012 / 01 / 20 \sim 02 / 03$ & 1 & 1 & 59 \\
\hline $2012 / 02 / 27 \sim 04 / 05$ & 19 & 3 & 235 \\
\hline $2012 / 06 / 29 \sim 07 / 19$ & 45 & 2 & 226 \\
\hline $2012 / 10 / 16 \sim 11 / 02$ & 3 & 1 & 87 \\
\hline $2014 / 08 / 26 \sim 09 / 27$ & 11 & 1 & 205 \\
\hline $2014 / 10 / 09 \sim 11 / 06$ & 49 & 6 & 209 \\
\hline $2014 / 11 / 01 \sim 11 / 15$ & 12 & 1 & 104 \\
\hline $2014 / 12 / 01 \sim 2015 / 01 / 12$ & 20 & 1 & 310 \\
\hline
\end{tabular}

lar flare activity, because the high energy neutrons could produce with high energy ions accelerated with the reconnection of magnetic field lines toward to the solar photosphere. The solar photosphere brightening after solar flare was observed in Active region(AR)1890 on November 8, 2013 at 4:23-5:40 UT associated with $\mathrm{H} \alpha$ variation as shown int Figure 3 The observed flare magnitude is reported as X1.1. Starting time,peak time and end time are 4:20, 4:26 and 4:29 respectively reported in GOES catalog. In our observations( Figure 4 both $\mathrm{H} \alpha$ and $\mathrm{CaK}$ imaging indicated large intensity variations above $5 \%$ order of intensity in 5 minutes before this flare activity. This could be the precursor of the flare explosion. Then $\mathrm{H} \alpha$ and $\mathrm{CaK}$ imaging data presented the sharp rising of the flare signals simultaneously, and $\mathrm{H} \alpha$ signals exponentially decayed in fifteen minutes, but $\mathrm{CaK}$ ones did in less than five minutes. The light curve variation in $\mathrm{CaK}$ images could predict the collisions of the ions in the arcade of large magnetic loop in the corona with the magnetic reconnecting force toward to the solar photosphere. However, the NM data[7] did not show any enhancements with this events.

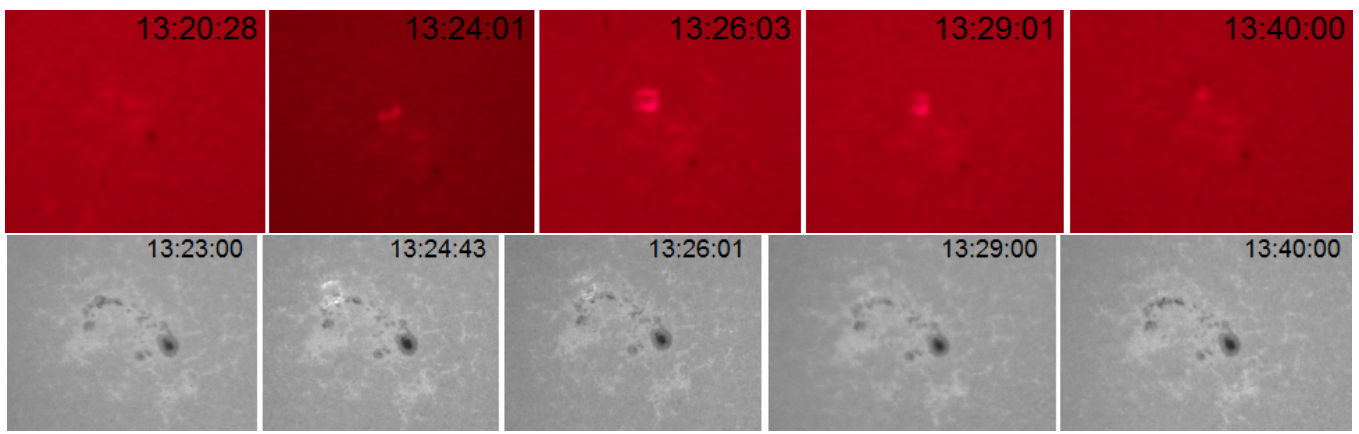

Figure 3: Time variation of AR1890 on 2013/11/08 JST13:23:00-14:40:00 with $\mathrm{H} \alpha$ (upper panel) and CaK imaging(lower panel) 

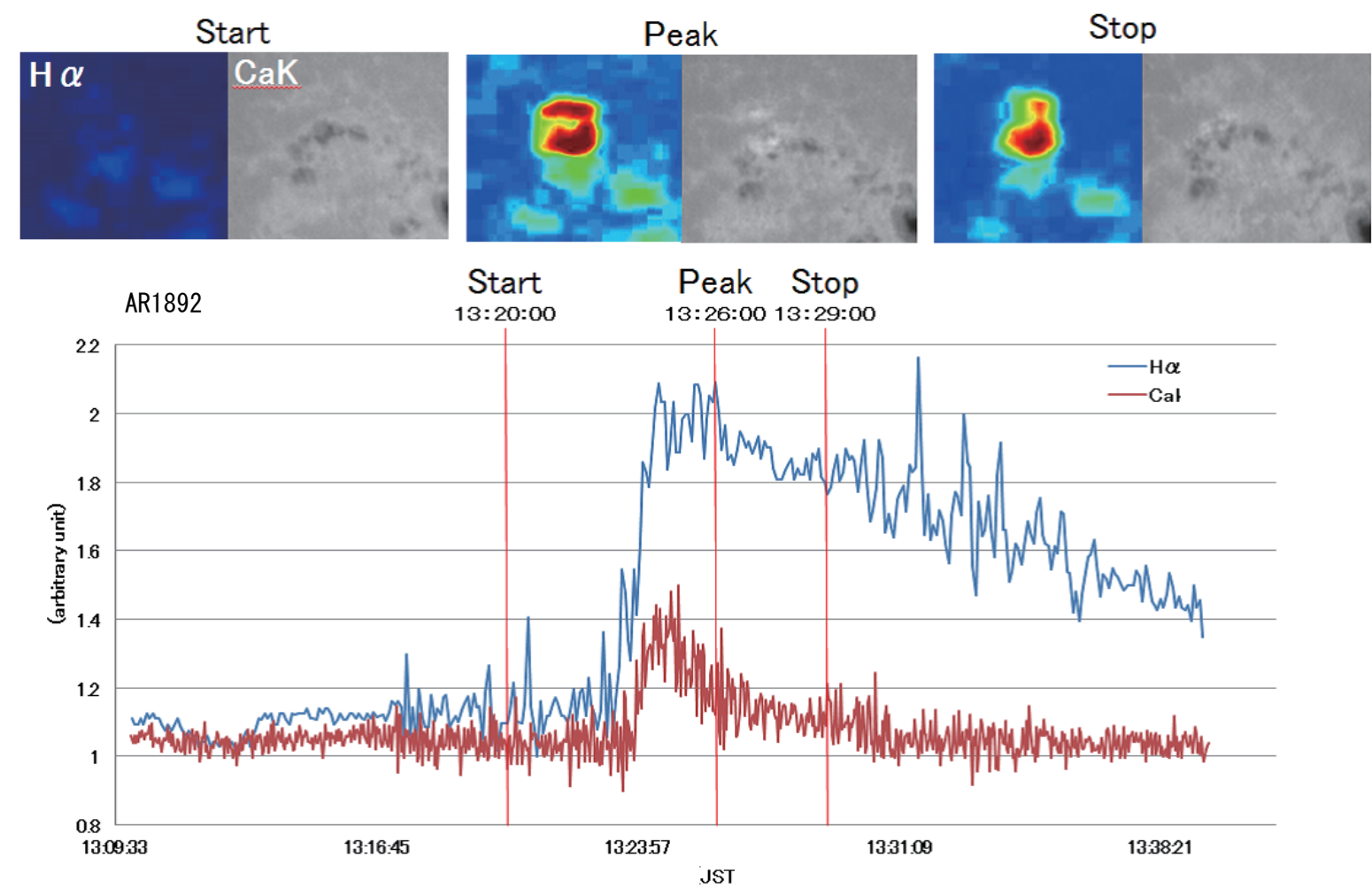

Figure 4: Light cure of $\mathrm{H} \alpha$ and $\mathrm{CaK}$ imaging of AR1892 when X1.1 flare occurring on 2013/11/08

The other results came from the OUS4 EAS array which observed low energy muons. In Figure 5 hourly cosmic ray muon rate were plotted with the colored chart of the solar activities such as solar flares of magnitude, CME events and interplanetary magnetic field variations observed at NICT from November 1, 2011 to November 14, 2011. The larges solar flare magnitude was X1.9 at November 3, 2011 associated with 10 M-class flares and $6 \mathrm{CME}$ events. No significant variation in the OUS4 EAS muon data have been observed during this period. The X 1.9 class flare, numbered AR1339, occurred on 11:27 in November 3, 2011 at the left side of the sun into Earth's view, and on the other hand, the CME took place on the backside of the sun at 14:35 in the same day.

One of the most significant solar flares occurred in March 7, 2012, numbered AR1429, at 00:24 UTC with multiple CME events. This event is the second largest solar flare event in Cycle 24. In Figure 6, the EAS OUS4 array has observed the Forbush decreasing of cosmic rays after about two days with $3 \sigma$ significances. This is the most clear events observed in the EAS OUS4 array muon monitoring system. The neutron monitor at Moscow reported about $10 \%$ decreases suddenly at March 8, 2012 and gradual recovering for two weeks after this sudden decrease.

The giant sunspot region appeared from October 22, 2014 of which size is comparable to Jupiter diameter and which is the largest active region in Cycle 24. This AR2912 unleashed six X-class flares from 19th to 28th of October 2014. The largest one is X3.1 magnitude on October 24, 2014. Including M-class flare, fifteen solar flares have been observed in the period. On the other hand, the only five CME events were identified. The cosmic ray hourly intensity variations 


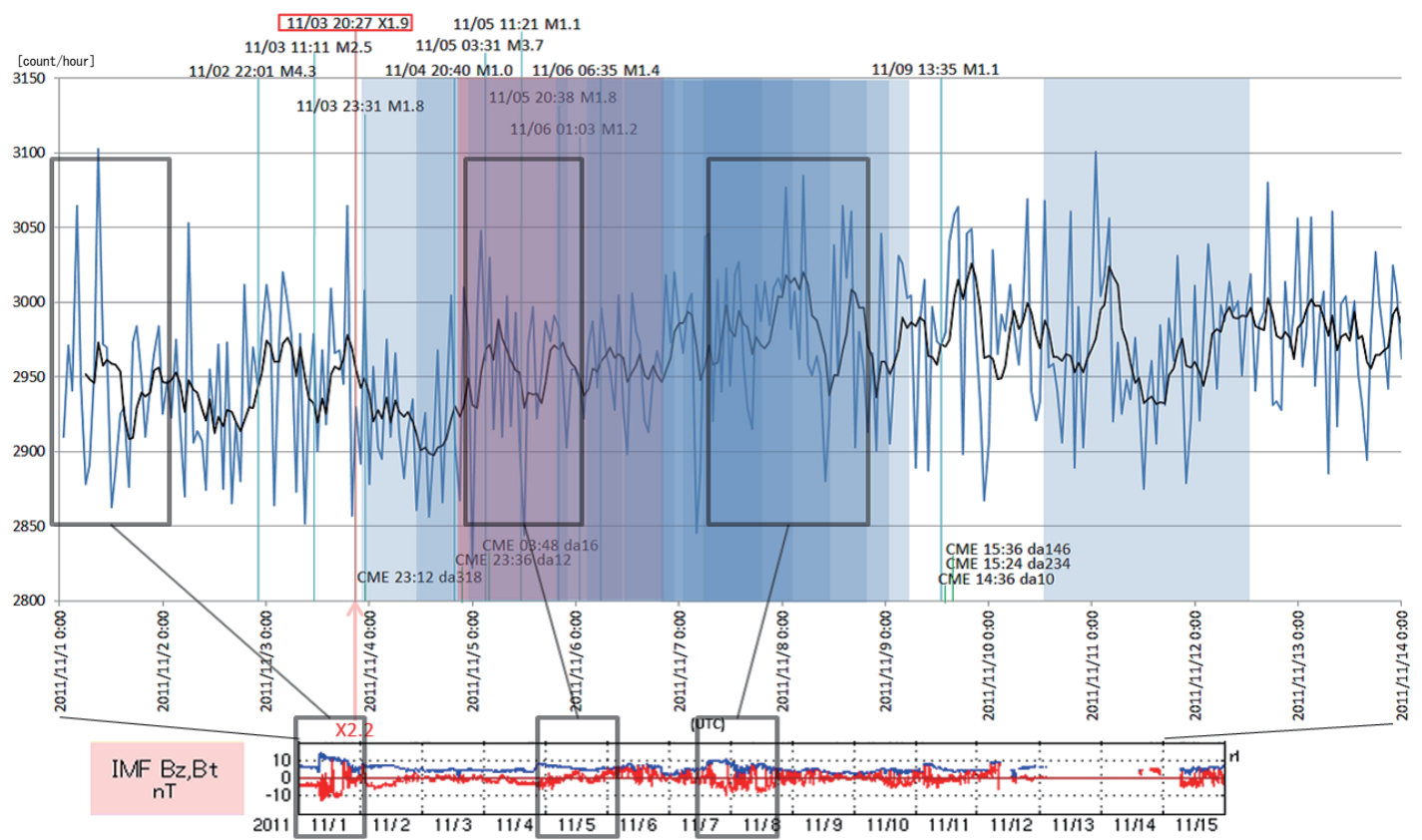

Figure 5: Cosmic ray intensity variation with solar events between 2011/11/1 11/14 with solar activities

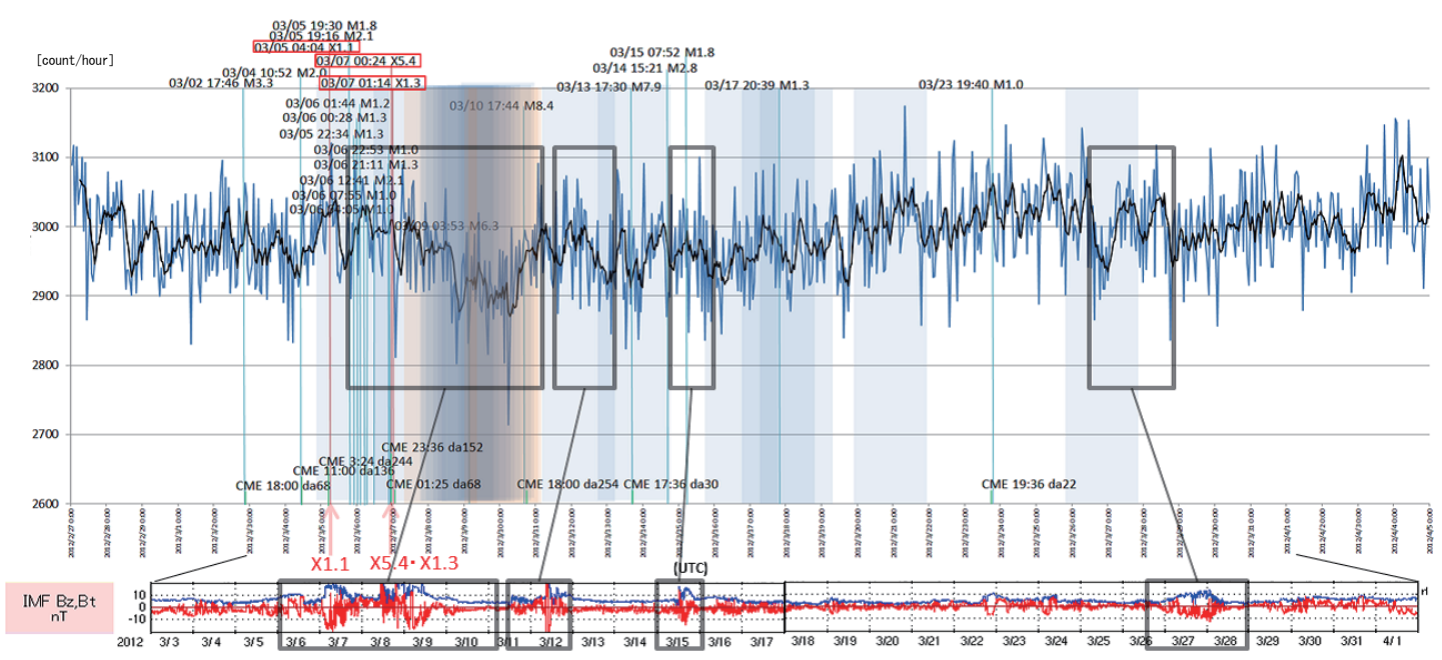

Figure 6: Cosmic ray intensity variation with solar events between 2012/2/27 4/5 with the solar activities

observed by EAS OUS4 array is shown in Figure 7 during the wider time span. The solar activities observed by $\mathrm{CaK}$ imaging did not detect any variation in the photosphere indeed. The hourly count rates almost seem to be explained within statistical fluctuations. This tendencies were also observed in the NM network at Moscow[7]. Although this huge active region AR2192 traveled across the sun facing to the Earth for 14 days, significant influence in the cosmic ray at the Earth was not observed. 


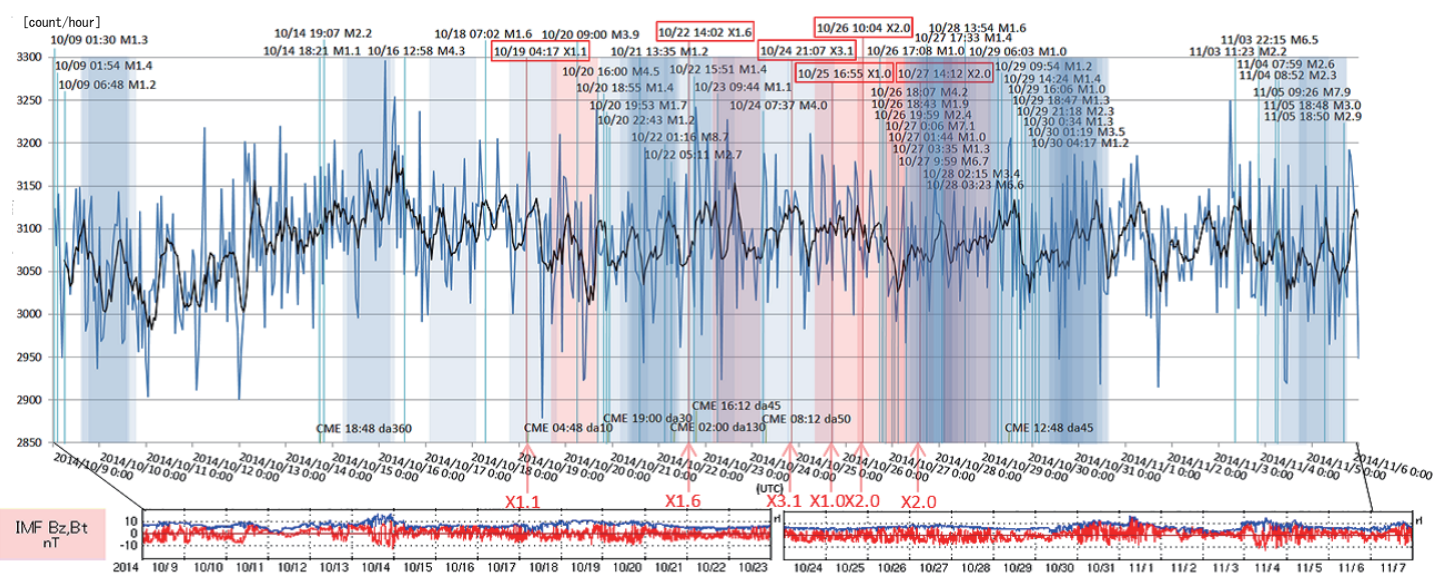

Figure 7: Cosmic ray intensity variation with solar events between 2014/10/09 11/06 with the solar activities on AR2192

\section{Summary}

The cosmic ray intensity variation analysis by using one of the LAAS EAS arrays has been performed for the part of the the 24th solar cycle. We have observed Forbush decreases in March 2012 due to AR1429 solar activities, but no signals have detected in the largest AR numbered 2912. In the observation of $\mathrm{H} \alpha$ and $\mathrm{CaK}$ telescope observations, we have carried out second order rate image acquisitions for the daytime. While data were limited by the weather condition because of the observation from the Earth's surface, we have detected CaK light curve time variation associated with the solar flare on November 11, 2013 at AR1892. This flare shows precursor signal fluctuations before five minutes of the flare start time. The CaK intensity enhancements after the flare have also been observed. We only have two events of $\mathrm{CaK}$ precursor and $\mathrm{CaK}$ image enhancement. We need more data and have to analyze the related data such as the neutron monitors and muon monitors as well as our EAS array muon data.

\section{References}

[1] Neutron Monitor Database-NMDB (http://cosrays.izmiran.ru/main.htm)

[2] S. E. Forbus ,On the Effects in Cosmic-Ray Intensity, Phys. Rev. 1937,51, 1108-1109

[3] E. L. Chupp et. al., A DIRECT OBSERVATION OF SOLAR NEUTRONS,Astrophys. J., 1982,263 L95-99

[4] A. Iyono et al., Cosmic ray composition studies, Astrophysics and Space Sciences Transactions, 2011, $7327-333$

[5] H. Matsumoto et. al., Simulation studies and implementation, Nucl. Instr. \& Meth. A, 2010, 614 475-482

[6] Y. Muraki et. al., Detection of high-energy solar neutron, Astropart. Phys., 2008, 29 229-242

[7] Neutron Monitor Database-NMDB (http://cosrays.izmiran.ru/main.htm) 